\title{
On an Open Problem of U. Höhle - A Characterization of Conditionally Cancellative T-subnorms
}

\author{
Balasubramaniam Jayaram \\ Indian Institute of Technology Hyderabad, \\ Yeddumailaram - 502 205, INDIA \\ jbala@iith.ac.in
}

\begin{abstract}
In this work we solve an open problem of U.Höhle [Problem 11, Fuzzy Sets and Systems 145 (2004) 471-479]. We show that the solution gives a characterization of all conditionally cancellative t-subnorms. Further, we give an equivalence condition for a conditionally cancellativite t-subnorm to be a t-norm and hence show that conditionally cancellativite t-subnorms whose natural negations are strong are, in fact, t-norms.
\end{abstract}

\section{Introduction}

The paper by Klement et al. [6] is a collection of open problems posed during the $24^{\text {th }}$ Linz Seminar on fuzzy set theory. They deal with unsolved problems (as of then) related to fuzzy aggregation operations, especially t-norms and tsubnorms. Since the publication of [6], some problems mentioned therein have been solved - for instance, Problem 1 was solved by Ouyang et al. [8], Problem $\mathbf{5}$ was solved by Ouyang and $\mathrm{Li}[8]$ while for some other problems partial solutions have been given, see for instance, the papers of Viceník [9], [10], [11] relating to Problem 4(i).

One of the open problems listed therein was posed by Prof. U. Höhle (Problem 11) which reads as follows:

Problem 1 (U.Höhle, [6], Problem 11). Characterize all left-continuous t-norms $T$ which satisfy

$$
I(x, T(x, y))=\max (n(x), y), \quad x, y \in[0,1] .
$$

where $I$ is the residual operator linked to $T$, i.e.,

$$
\begin{aligned}
I(x, y) & =\sup \{t \in[0,1] \mid T(x, t) \leq y\}, \quad x, y \in[0,1], \\
n(x) & =n_{T}(x)=I(x, 0) \text { for all } x \in[0,1] .
\end{aligned}
$$

Further, Prof. U.Höhle goes on to remark the following:

Remark 1. "In the class of continuous t-norms, only nilpotent t-norms fulfill the above property." 
In this work we deal with two problems. Firstly, we solve the above open problem of U.Höhle and show that the solution gives a characterization of all conditionally cancellative t-subnorms. From the proven result it does follow that the remark of Prof. U.Höhle - Remark 1 - is not always true and give an equivalence condition for it to be true, viz., that the natural negation obtained from the t-norm is strong.

Secondly, this quite naturally leads us to consider conditionally cancellative t-subnorms whose natural negations are involutive. Once again, by proving an equivalence condition for a conditionally cancellative t-subnorm to be a t-norm, we show that conditionally cancellative t-subnorms whose natural negations are involutive, in fact, become t-norms.

\section{Preliminaries}

Definition 1. A function $N:[0,1] \rightarrow[0,1]$ is called a fuzzy negation if $N$ is decreasing and $N(0)=1, N(1)=0$.

Definition 2 ([5], Definition 1.7). A t-subnorm is a function $M:[0,1]^{2} \rightarrow$ $[0,1]$ such that it is monotonic non-decreasing, associative, commutative and $M(x, y) \leq \min (x, y)$ for all $x, y \in[0,1]$.

Note that for a t-subnorm 1 need not be the neutral element, unlike in the case of a t-norm.

Definition 3 (cf. [5], Definition 2.9 (iii)). A t-subnorm $M$ satisfies the Conditional Cancellation Law if, for any $x, y, z \in(0,1]$,

$$
M(x, y)=M(x, z)>0 \text { implies } y=z .
$$

Alternately, (CCL) implies that on the positive domain of $M$, i.e., on the set $\left\{(x, y) \in(0,1]^{2} \mid M(x, y)>0\right\}, M$ is strictly increasing.

Definition 4 (cf. [1], Definition 2.3.1). Let $M$ be any t-subnorm. Its natural negation $n_{M}$ is given by

$$
n_{M}(x)=\sup \{t \in[0,1] \mid M(x, t)=0\}, \quad x \in[0,1] .
$$

Note that though $n_{M}(0)=1$, it need not be a fuzzy negation, since $n_{M}(1)$ can be greater than 0 . However, we have the following result.

Lemma 1 (cf. [1], Proposition 2.3.4). Let $M$ be any $t$-subnorm and $n_{M}$ its natural negation. Then we have the following:

(i) $M(x, y)=0 \Longrightarrow y \leq n_{M}(x)$.

(ii) $y<n_{M}(x) \Longrightarrow M(x, y)=0$.

(iii) If $M$ is left-continuous then $y=n_{M}(x) \Longrightarrow M(x, y)=0$, i.e., the reverse implication of (i) also holds. 


\section{Solution to the Open Problem of U. Höhle}

It should be noted that in the case $T$ is left-continuous - as stated in Problem 1 - the sup in (2) actually becomes max. It is worth mentioning that the residual can be determined for more generalised conjunctions and the conditions underwhich this residual becomes a fuzzy implication can be found in, for instance, [2], [4]. Hence we further generalise the statement of Problem $\mathbf{1}$ by considering a t-subnorm instead of a t-norm and also dropping the condition of left-continuity. As we show below the solution characterizes the set of all conditionally cancellative t-subnorms.

Theorem 1. Let $M$ be any t-subnorm and $I$ the residual operation linked to $M$ by (2). Then the following are equivalent:

(i) The pair $(I, M)$ satisfies (1).

(ii) $M$ is a Conditionally Cancellative t-subnorm.

Proof. Let $M$ be any t-subnorm, not necessarily left-continuous. Note that we denote $n_{M}$ simply by $n$.

(i) $\Longrightarrow$ (ii): Let the adjoint pair $(I, M)$ satisfy (1). On the contrary, let us assume that there exist $x, y, z \in(0,1)$ such that $M(x, y)=M(x, z)>0$ but $y<z$. Then we have that

$$
\operatorname{LHS}(1)=I(x, M(x, y))=\sup \{t \in[0,1] \mid M(x, t) \leq M(x, y)\} \geq z>y .
$$

However, note that, from Lemma 1 (i) we have that $y \geq n(x)$, since $M(x, y)>$ 0 . Thus

$$
\operatorname{RHS}(1)=\max (n(x), y)=y<\operatorname{LHS}(1)
$$

a contradiction to the fact that the adjoint pair $(I, M)$ satisfies (1). Hence $M$ satisfies (CCL).

(ii) $\Longrightarrow($ i): Now, let $M$ satisfy (CCL). Consider any arbitrary $x, y \in[0,1]$. Then either $n(x)>y$ or $n(x) \leq y$.

If $n(x)>y$, then by Lemma 1 (ii) we see that $M(x, y)=0$ and hence

$$
\text { LHS }(1)=I(x, M(x, y))=I(x, 0)=n(x)=\max (n(x), y)=\operatorname{RHS}(1) .
$$

If $n(x) \leq y$ and $M(x, y)=0$ then by Lemma $1(\mathrm{i})$ we have that $n(x) \geq y$ and hence $n(x)=y$ and it reduces to the above case. Hence let $M(x, y)>0$. Then

$$
\operatorname{RHS}(1)=\max (n(x), y)=y \text {. }
$$

We claim now that LHS $(1)=I(x, M(x, y))=y$. If this were not true, then there exists $1 \geq z>y(z \nless y$ by the monotonicity of $M)$ such that

$$
I(x, M(x, y))=\sup \{t \in[0,1] \mid M(x, t) \leq M(x, y)\}=z .
$$


This implies that there exists a $w \in(0,1)$ such that $z>w>y$ and $M(x, w) \leq M(x, y)$, which by the monotonicity of t-subnorm implies that $M(x, w)=M(x, y)$ with $w \geqslant y$, a contradiction to the fact that $M$ satisfies (CCL). Thus the adjoint pair $(I, M)$ satisfies (1).

Example 1. Consider the product t-norm $T_{\mathbf{P}}(x, y)=x y$, which is a strict $\mathrm{t}$ norm and hence continuous and Archimedean, whose residual is the Goguen implication given by

$$
I_{\mathrm{GG}}(x, y)= \begin{cases}1, & \text { if } x \leq y \\ \frac{y}{x}, & \text { if } x>y\end{cases}
$$

It can be easily verified that the pair $\left(T_{\mathbf{P}}, I_{\mathbf{G G}}\right)$ does indeed satisfy $(1)$ whereas the natural negation of $T_{\mathbf{P}}$ is the Gödel negation

$$
n_{T_{\mathbf{P}}}(x)=I_{\mathbf{G G}}(x, 0)= \begin{cases}1, & \text { if } x=0, \\ 0, & \text { if } x>0 .\end{cases}
$$

This example clearly shows that the remark of U.Höhle, Remark 1, is not always true. In the following we give an equivalence condition under which it is true.

Theorem 2. Let $T$ be a continuous t-norm that satisfies (1) along with its residual. Then the following are equivalent:

(i) $T$ is nilpotent.

(ii) $n_{T}$ is strong.

Proof. (i) $\Longrightarrow$ (ii): Obvious.

(ii) $\Longrightarrow$ (ii): If $T$ is continuous and satisfies (1) along with its residual then, from Theorem 1, $T$ is conditionally cancellative and hence necessarily Archimedean by [5], Proposition 2.15 (ii). Thus $T$ is either nilpotent or strict. If $T$ is continuous with a strong natural negation, clearly, $T$ has zero-divisors and hence $T$ is nilpotent.

\section{Conditional Cancellativity and Unit element}

From the above remarks we note that when the natural negation of the underlying conjunction (a continuous t-norm, in the above case) is strong the class of conjunctions that satisfy (1) along with its residual gets restricted. Hence we study the class of t-subnorms $M$ that satisfy (1) along with its residual and whose natural negations are strong. In other words, we seek the characterization of the class of conditionally cancellative t-subnorms with strong natural negations.

Let us recall from the remark following Definition 4 that the natural negation of a t-subnorm $n_{M}$ need not be a fuzzy negation. If a t-subnorm has 1 as its neutral element, i.e., if it is a t-norm, then we have

$$
\begin{aligned}
M(1, y) & =0 \Longleftrightarrow y=0, \\
\text { i.e., } y & =\sup \{t \mid M(1, t)=0\}=n_{M}(1)=0 .
\end{aligned}
$$


Equivalently, by the monotonicity of $M$ we have that $n_{M}$ is a fuzzy negation. However, this is only a necessary and not a sufficient condition.

Note that, so far, no general result giving equivalence conditions under which a t-subnorm becomes a t-norm is available. It was Jenei [3] who proposed some suficiency conditions and showed that left-continuous t-subnorms with strong natural negations are t-norms, i.e., 1 does become a neutral element.

In the following we give an equivalence condition for a conditionally cancellative t-subnorm to be a t-norm and show that in the case $n_{M}$ is a strong negation then $M$ always is a t-norm.

Lemma 2. Let $M$ be a conditionally cancellative t-subnorm. Let $M\left(1, y_{0}\right)=y_{0}$, for some $y_{0} \in(0,1]$.

(i) Then $M(1, y)=y$ for all $y \in\left[y_{0}, 1\right]$.

(ii) Let $y^{*}=\sup \{t \mid M(1, t)=0\}=n_{M}(1)$. Then $M(1, y)=y$ for all $y \in\left(y^{*}, y_{0}\right]$.

Proof. Let $M\left(1, y_{0}\right)=y_{0}$, for some $y_{0} \in(0,1]$.

(i) Let $y_{0}<y \leq 1$. Clearly, $y_{0}=M\left(1, y_{0}\right)<M(1, y) \leq y$. If $M(1, y)=y^{\prime}<y$, then by associativity and conditional cancellativity we have

$$
\left.\begin{array}{l}
M\left(M\left(1, y_{0}\right), y\right)=M\left(y_{0}, y\right) \\
M\left(M(1, y), y_{0}\right)=M\left(y^{\prime}, y_{0}\right)
\end{array}\right\} \Longrightarrow M\left(y_{0}, y\right)=M\left(y_{0}, y^{\prime}\right) \Longrightarrow y=y^{\prime},
$$

i.e., $M(1, y)=y$ for all $y \geq y_{0}$.

(ii) Let $y^{*}<y \leq y_{0}$. Clearly, $y_{0}=M\left(1, y_{0}\right)>y \geq M(1, y)=y^{\prime}$. If $M(1, y)=$ $y^{\prime}<y$, then, once again, by associativity and conditional cancellativity we have

$$
\left.\begin{array}{l}
M\left(M\left(1, y_{0}\right), y\right)=M\left(y_{0}, y\right) \\
M\left(M(1, y), y_{0}\right)=M\left(y^{\prime}, y_{0}\right)
\end{array}\right\} \Longrightarrow M\left(y_{0}, y\right)=M\left(y_{0}, y^{\prime}\right) \Longrightarrow y=y^{\prime},
$$

i.e., $M(1, y)=y$ for all $y \in\left(y^{*}, y_{0}\right]$.

Based on the above result, we now have the following equivalence condition for a conditionally cancellative t-subnorm to be a t-norm:

Theorem 3. Let $M$ be any conditionally cancellative $t$-subnorm. Then the following are equivalent:

(i) $M$ is a t-norm.

(ii) $n_{M}$ is a negation and $M\left(1, y_{0}\right)=y_{0}$, for some $y_{0} \in(0,1]$.

Proof. Sufficiency is obvious. Necessity follows from the fact that if $n_{M}$ is a negation then $y^{*}=0$ in Lemma 2 above.

The final result of this work shows that in the case $n_{M}$ is a strong negation then $M$ always is a t-norm. 
Theorem 4. Let $M$ be any conditionally cancellative t-subnorm. If $n_{M}$ is a strong natural negation then $M$ is a t-norm.

Proof. Our approach will be to show that $M(1,1)=1$ and then the result follows easily from Theorem 3 . Note also that since $n_{M}$ is a strong negation, we have that $n_{M}(x)=1 \Longleftrightarrow x=0$ and $n_{M}(x)=0 \Longleftrightarrow x=1$. Equivalently, $M(1, x)=0 \Longleftrightarrow x=0$.

On the contrary, let us assume that $M(1, y)<y$ for all $y \in(0,1]$. In particular, $M(1,1)=z$ such that $0<z<1$. Since $n_{M}$ is strong, there exists a $z^{\prime} \in(0,1)$ such that $z=n_{M}\left(z^{\prime}\right)$. We claim that $z^{\prime}=0$ and hence $z=1$.

If not, then there exists $0<z^{\prime \prime}<z^{\prime}$ and by the definition of $n_{M}$ we have that $M\left(z, z^{\prime \prime}\right)=0$. Also, by our assumption $0<M\left(1, z^{\prime \prime}\right)=z^{*}<z^{\prime \prime}$. Now, by associativity and conditional cancellativity we have

$$
\begin{aligned}
& \left.\begin{array}{l}
M\left(M(1,1), z^{\prime \prime}\right)=M\left(z, z^{\prime \prime}\right) \\
M\left(M\left(1, z^{\prime \prime}\right), 1\right)=M\left(z^{*}, 1\right)
\end{array}\right\} \Longrightarrow M\left(z, z^{\prime \prime}\right)=0=M\left(z^{*}, 1\right) \\
& \Longrightarrow z^{*}=0,
\end{aligned}
$$

a contradiction. Thus $z=1$ and hence we have the result.

\section{Concluding Remarks}

In this work we have solved a more generalised version of an open problem of U.Höhle and shown that the solution gives a characterization of all conditionally cancellative t-subnorms. Further, by proving an equivalence condition for a conditionally cancellative t-subnorm to be a t-norm, we have shown that conditionally cancellative t-subnorms with involutive natural negations are t-norms.

\section{References}

1. Baczyński M., Jayaram B. (2008) Fuzzy Implications. Vol. 231, Studies in Fuzziness and Soft Computing, Springer-Verlag, Heidelberg.

2. Demirli K., B. De Baets (1999) Basic properties of implicators in a residual framework, Tatra Mount. Math. Publ. 16:31-46.

3. Jenei S. (2001) Continuity of left-continuous triangular norms with strong induced negations and their boundary condition. Fuzzy Sets and Systems 124:35-41.

4. Jayaram B., Mesiar R. (2009) I-Fuzzy Equivalence Relations and I-Fuzzy Partitions Info Sci. 179:1278-1297.

5. Klement E.P., Mesiar R., Pap E. (2000) Triangular norms. Kluwer, Dordrecht.

6. Klement E.P., Mesiar R., Pap E. (2004) Problems on triangular norms and related operators. Fuzzy Sets and Systems 145:471-479.

7. Ouyang Y., J. Li, J. Fang (2006) A conditionally cancellative left-continuous tnorm is not necessarily continuous. Fuzzy Sets and Systems 157:2328-2332.

8. Ouyang Y., J. Li (2005) An answer to an open problem on triangular norms. Info Sci. 175:78-84.

9. Viceník P. (2005) Additive generators of associative functions. Fuzzy Sets and Systems 153:137-160. 
10. Viceník P. (2008) Intersections of ranges of additive generators of associative functions. Tatra Mt. Math. Publ. 40:117-131

11. Viceník P. (2008) Additive generators of border-continuous triangular norms. Fuzzy Sets and Systems 159:1631-1645. 